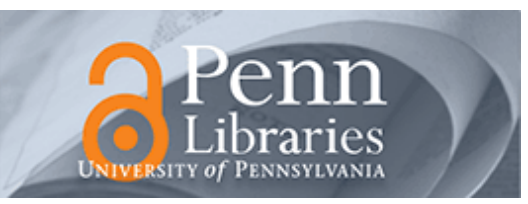

University of Pennsylvania

ScholarlyCommons

9-16-2009

\title{
Vector Correlation Technique for Pixel-wise Detection of Collagen Fiber Realignment During Injurious Tensile Loading
}

Kyle P. Quinn

University of Pennsylvania, kpquinn@seas.upenn.edu

Beth A. Winkelstein

University of Pennsylvania, winkelst@seas.upenn.edu

Follow this and additional works at: https://repository.upenn.edu/be_papers

Part of the Biomedical Engineering and Bioengineering Commons

\section{Recommended Citation}

Quinn, K. P., \& Winkelstein, B. A. (2009). Vector Correlation Technique for Pixel-wise Detection of Collagen Fiber Realignment During Injurious Tensile Loading. Retrieved from https://repository.upenn.edu/

be_papers/172

Suggested Citation: Quinn, K.P. and Winkelstein, B.A. (2009). "Vector correlation technique for pixel-wise detection of collagen fiber realignment during injurious tensile loading." Journal of Biomedical Optics. Vol. 14(5). 054010.

(c) 2009 Society of Photo-Optical Instrumentation Engineers. One print or electronic copy may be made for personal use only. Systematic reproduction and distribution, duplication of any material in this paper for a fee or for commercial purposes, or modification of the content of the paper are prohibited.

http://dx.doi.org/10.1117/1.3227037

This paper is posted at ScholarlyCommons. https://repository.upenn.edu/be_papers/172

For more information, please contact repository@pobox.upenn.edu. 


\title{
Vector Correlation Technique for Pixel-wise Detection of Collagen Fiber Realignment During Injurious Tensile Loading
}

\begin{abstract}
Excessive soft tissue loading can produce adverse structural and physiological changes in the absence of any visible tissue rupture. However, image-based analysis techniques to assess microstructural changes during loading without any visible rupture remain undeveloped. Quantitative polarized light imaging (QPLI) can generate spatial maps of collagen fiber alignment during loading with high temporal resolution and can provide a useful technique to measure microstructural responses. While collagen fibers normally realign in the direction that tissue is loaded, rapid, atypical fiber realignment during loading may be associated with the response of a local collagenous network to fiber failure. A vector correlation technique was developed to detect this atypical fiber realignment using QPLI and mechanical data collected from human facet capsular ligaments $(n=16)$ loaded until visible rupture. Initial detection of anomalous realignment coincided with a measurable decrease in the tissue stiffness in every specimen and occurred at significantly lower strains than those at visible rupture $(\rho<0.004)$, suggesting this technique may be sensitive to a loss of microstructural integrity. The spatial location of anomalous realignment was significantly associated with regions where visible rupture developed $(\rho<0.001)$. This analysis technique provides a foundation to identify regional differences in soft tissue injury tolerances and relevant mechanical thresholds.
\end{abstract}

\section{Keywords}

collagen finer, vector correlation, polarized light, damage, image analysis

\section{Disciplines}

Biomedical Engineering and Bioengineering | Engineering

\section{Comments}

Suggested Citation: Quinn, K.P. and Winkelstein, B.A. (2009). "Vector correlation technique for pixel-wise detection of collagen fiber realignment during injurious tensile loading." Journal of Biomedical Optics. Vol. 14(5). 054010.

(c) 2009 Society of Photo-Optical Instrumentation Engineers. One print or electronic copy may be made for personal use only. Systematic reproduction and distribution, duplication of any material in this paper for a fee or for commercial purposes, or modification of the content of the paper are prohibited.

http://dx.doi.org/10.1117/1.3227037 


\section{Vector correlation technique for pixel-wise detection of collagen fiber realignment during injurious tensile loading}

Kyle P. Quinn

Beth A. Winkelstein

University of Pennsylvania

Department of Bioengineering

Spine Pain Research Laboratory and

Department of Neurosurgery

240 Skirkanich Hall

210 South 33rd Street

Philadelphia, Pennsylvania 19104

\begin{abstract}
Excessive soft tissue loading can produce adverse structural and physiological changes in the absence of any visible tissue rupture. However, image-based analysis techniques to assess microstructural changes during loading without any visible rupture remain undeveloped. Quantitative polarized light imaging (QPLI) can generate spatial maps of collagen fiber alignment during loading with high temporal resolution and can provide a useful technique to measure microstructural responses. While collagen fibers normally realign in the direction that tissue is loaded, rapid, atypical fiber realignment during loading may be associated with the response of a local collagenous network to fiber failure. A vector correlation technique was developed to detect this atypical fiber realignment using QPLI and mechanical data collected from human facet capsular ligaments $(n=16)$ loaded until visible rupture. Initial detection of anomalous realignment coincided with a measurable decrease in the tissue stiffness in every specimen and occurred at significantly lower strains than those at visible rupture $(p<0.004)$, suggesting this technique may be sensitive to a loss of microstructural integrity. The spatial location of anomalous realignment was significantly associated with regions where visible rupture developed $(p<0.001)$. This analysis technique provides a foundation to identify regional differences in soft tissue injury tolerances and relevant mechanical thresholds. ๑ 2009 Society of Photo-Optical Instrumentation Engineers. [DOI: 10.1117/1.3227037]
\end{abstract}

Keywords: collagen fiber; vector correlation; polarized light; damage; image analysis.

Paper 09195R received May 14, 2009; revised manuscript received Jul. 2, 2009 ; accepted for publication Jul. 21, 2009; published online Sep. 16, 2009.

\section{Introduction}

Tensile loading of ligament and tendon tissue can induce collagen fiber disorganization, ${ }^{1,2}$ fibroblast necrosis, ${ }^{3}$ and nociceptor activation ${ }^{4}$ in the absence of visible tissue rupture and before catastrophic tissue failure. However, conventional imaging techniques, such as computed tomography or magnetic resonance imaging, often cannot identify less severe tissue damage that can be sustained during ligament sprains and other painful injuries. ${ }^{5-7}$ Historically, biomechanical studies of tissue injury have been primarily focused on defining ligament tolerances at mechanical failure, where tissue rupture is unmistakably detectable by visual inspection. ${ }^{7-9}$ Although these traditional methods of defining tissue tolerance and identifying the location of injury have provided valuable advances in injury prevention, detecting the initiation of mechanical damage during soft tissue loading, and localizing it spatially, is necessary to begin to develop both physiologically relevant injury tolerances and an anatomical understand-

Address all correspondence to: Beth A. Winkelstein, PhD, Department of Bioengineering, University of Pennsylvania, 240 Skirkanich Hall, 210 South 33rd Street, Philadelphia, PA 19104-6321. Tel: 215-573-4589; Fax: 215-5732071; E-mail: winkelst@seas.upenn.edu ing of the mechanical manifestations of tissue damage.

Polarized light imaging, ${ }^{10,11}$ optical coherence tomography, ${ }^{12}$ confocal microscopy, ${ }^{13,14}$ and small-angle light scattering ${ }^{15}$ techniques have all been used to quantify microstructural changes in soft tissue during mechanical loading. However, of these, quantitative polarized light imaging (QPLI) has been used to infer collagen fiber kinematics through the thickness of soft tissues in all regions during continuous loading because of its ability to acquire data with high temporal resolution and adjustable spatial resolution. ${ }^{10,16} \mathrm{Be}-$ cause of these advantages, QPLI studies of ligaments and engineered constructs have identified local changes in collagen fiber kinematics, which are associated with modifications in mechanical performance under tensile loading prior to gross tissue failure. ${ }^{10,17}$ Strain measurements have been used previously to define tissue responses and injury thresholds in a variety of studies of soft tissue injury and damage. ${ }^{4,18-22}$ Yet, the location of microstructural damage in the tissue may not directly correspond to the location with the maximum principal strain. ${ }^{17}$ With an apparent disconnect between tissue strain measurements and the location of tissue damage, there is an

1083-3668/2009/14(5)/054010/10/\$25.00 @ 2009 SPIE 
incomplete understanding of both where microstructural damage initiates in tissue and the mechanical conditions necessary to produce this damage prior to visible rupture of the tissue structure.

Although QPLI techniques can define both the mean fiber direction and the strength of the alignment of collagen fibers in soft tissues at every pixel for which polarized light data are acquired, ${ }^{10,16,23,24}$ analysis techniques to detect any microstructural damage in ligament tissue using QPLI or any other nondestructive imaging technique remain largely rudimentary. These previous analysis techniques made advances toward the identification of fiber kinematics during loading that were associated with mechanical failure. ${ }^{10,17}$ However, in those approaches, it was necessary to average the alignment data over tissue regions of a size sufficient to track their change in alignment over time. With the localization of altered fiber kinematics and damage restricted to large predefined tissue regions in those studies, ${ }^{10,17}$ any microstructural damage could go undetected if it occurred in only a small fraction of a discretized region. Thus, an analysis technique such as image correlation, ${ }^{25-27}$ which does not require any marker tracking or pixel-binning into large tissue regions, is needed both for analyzing the full pixelwise resolution of QPLI alignment data acquired over an image space and for accurately detecting the occurrence of microstructural damage and its precise location within a tissue.

The goal of this study was to develop a novel analysis technique to nondestructively localize the occurrence of microstructural damage in ligament tissue during loading with pixelwise resolution using QPLI fiber alignment data and vector correlation techniques. The overall premise of this study is that microstructural damage develops in collagenous soft tissue when the load-bearing collagen fibers break under tension, and that failure of those collagen fibers requires that the tensile forces they supported be redistributed to the surrounding intact fibers. As a consequence of bearing this redistributed load, we propose that the local fiber network realigns substantially more than it normally does during loading without any fiber failures or associated redistribution of load. On the basis of this premise, we developed a method to identify any of this "anomalous" fiber realignment during continuous loading of capsular ligament tissue to visible rupture. This assessment of fiber realignment was performed by pixelwise vector correlation calculations between sequential fiber alignment maps obtained during loading; a vector correlation measurement that has been previously used to compare maps of wind velocity ${ }^{25,28}$ was adapted to compute a correlation value at each pixel using both the fiber direction and the strength of fiber alignment. Although this study utilizes QPLI to provide fiber alignment data, the vector correlation technique described can be applied to any imaging modality capable of generating maps of fiber alignment in collagenous tissue during or following mechanical loading. Given the physiological $^{3,4,29}$ and structural changes ${ }^{1,2,17}$ that can occur in ligaments and tendons without any visible tissue rupture, we propose that using this analysis method, anomalous fiber realignment in the ligament can be detected prior to any visible tissue rupture. Accordingly, this technique would provide a lower bound for mechanical tolerance in ligament tissue with greater relevance to potential physiologic dysfunction than tolerances defined by mechanical failure or visible tissue rup-
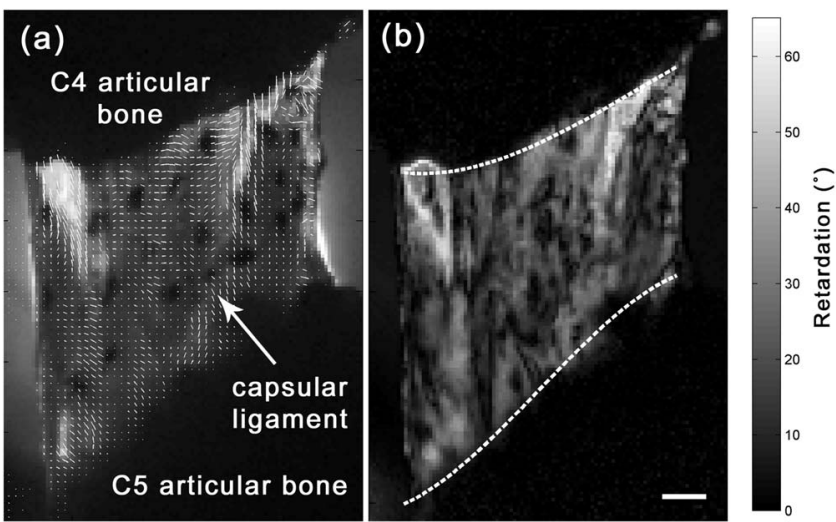

Fig. 1 Fiber alignment (a) direction and (b) retardation of specimen 4 at $1.49 \mathrm{~mm}$ of displacement. (a) Alignment vectors are plotted over the bright field image. The orientation of each vector represents the mean fiber direction at that pixel, and its length is scaled to the degree of retardation. (b) Pixel-wise retardation values vary from 0 to $65 \mathrm{deg}$ across this specimen. Retardation is the product of the tissue thickness and birefringence, and represents the strength of fiber alignment at a given pixel. The dotted lines represent the digitized ligament insertion boundaries, and the scale bar in (b) applies to both images and corresponds to $1 \mathrm{~mm}$.

ture. The onset and location of a decrease in the correlation between fiber alignment maps were compared to mechanical metrics of damage, tissue strain values, and visual inspection of the tissue integrity in order to provide mechanical context and validation of this methodology to detect putative microstructural damage through anomalous fiber realignment.

\section{Methods}

\subsection{Cadaveric Ligament Specimen Preparation}

Right and left cervical facet joints $(n=16)$ were removed from C4/C5 spinal motion segments of fresh, unembalmed human cadavers $(57 \pm 13$ years of age). The lateral aspect of the facet capsular ligament was isolated, and the articular processes of the joint were removed to allow for transmission of polarized light through the ligament tissue (Fig. 1). The boneligament-bone specimens were cast with FlowStone (Whip Mix Corporation, Louisville, Kentucky) in aluminum cups that were then fixed to an Instron 5385 testing machine (Instron Corporation, Norwood, Massachusetts). An array of 15-24 (mean 18.9 \pm 2.9 ) fiduciary marks was placed covering the ligament midsubstance surface using a felt-tipped pen in order to track tissue displacement for macroscale strain-field measurements during loading. The cross-sectional area of each unloaded specimen was estimated by measuring the sample's width and thickness with digital calipers to estimate tissue stress during loading. To reduce variability between specimen tests, the reference position for the start of loading was set at a $5-\mathrm{kPa}$ prestress based on the lowest measurable stress within the accuracy of our load cell. All specimens were mechanically preconditioned prior to loading for 30 cycles between 0 and $0.5 \mathrm{~mm}$, which corresponds to $<5 \%$ of the average load to failure. ${ }^{17}$ 


\subsection{Polarized Light Imaging Equipment and Theory}

Collagen fiber alignment was measured using a quantitative polarized light imaging system that has been previously described; ${ }^{10,17}$ the system generated pixelwise fiber-alignment maps throughout the entire period of mechanical loading of the specimen. The optical train of this system consisted of a rotating linear polarizer, the tissue sample, and a circular analyzer. The light source was provided by a fiber-optic illuminator with a focusing lens (Edmund Optics Inc., Barrington, New Jersey) and was transmitted through a $20-\mathrm{cm}$ rotating linear polarized disk (Edmund Optics Inc.) that was driven by a NEMA 17 stepper motor system (Lin Engineering, Santa Clara, California). A circular analyzer consisting of a Mica quarter-wave plate (Optosigma Corp., Santa Ana, California) and linear polarizing film (Edmund Optics Inc.) was mounted to a 6X macrozoom lens and a high-speed Phantom v5.1 CCD camera (Vision Research Inc., Wayne, New Jersey). A second Phantom (v4.3) camera recorded the orientation of the rotating polarizer; this measurement was used in the calculation of sample fiber direction. Both cameras were triggered to begin collecting data prior to specimen loading and were synchronized with the collection of the mechanical data through the Instron.

Under the assumption that ligament tissue is a linear birefringent material, ${ }^{30}$ the intensity of light $(I)$ measured by the CCD camera at any given pixel can be described as

$$
I(\theta)=\frac{1}{2} I_{\mathrm{o}}[\sin (\delta) \sin (2 \theta-2 \alpha)+1],
$$

where $I_{\mathrm{o}}$ is the initial intensity of the light source, $\delta$ is the retardation, $\theta$ is the angle of the rotating linear polarizer, and $\alpha$ is the sample's mean fiber-alignment direction. ${ }^{30}$ The retardation $(\delta)$ is taken as a measure of the strength of fiber alignment, or anisotropy, through the tissue thickness. ${ }^{10}$ Therefore, as the axis of the linear polarizer undergoes a complete 180-deg rotation, the intensity of light can be fit to the following simple harmonic equation:

$$
I(\theta)=A+B \cos (2 \theta)+C \sin (2 \theta) .
$$

As in previous studies, the Fourier coefficients, $A, B$, and $C$, were determined on a pixelwise basis using a summation approximation of the intensity data at discrete time points over the 180 -deg rotation. ${ }^{10,23}$ The signed harmonic coefficients, $B$ and $C$, were scaled by pixel intensity and used to calculate the retardation $(\delta)$ and the fiber-alignment direction $(\alpha)$ at each pixel using the following equations: ${ }^{10}$

$$
\begin{gathered}
\delta=\cos ^{-1}\left(\sqrt{1-B^{2}-C^{2}}\right), \\
\alpha=\frac{1}{2} \tan ^{-1}\left(\frac{B}{-C}\right) .
\end{gathered}
$$

\subsection{Specimen Testing and Data Acquisition}

For each test, the inferior articular facet of $\mathrm{C} 4$ was displaced at $0.5 \mathrm{~mm} / \mathrm{s}$ to impose tension across the C4/C5 capsular ligament until complete rupture of the ligament was produced. During each test, load and displacement data were collected by the Instron Bluehill software at $1 \mathrm{kHz}$ and the CCD cameras collected images with a $196 \times 400$ pixel window, at $500 \mathrm{fps}$ with $12.5 \mathrm{pixel} / \mathrm{mm}$ resolution. The linear polarizer rotated at $750 \mathrm{rpm}$, which corresponded to $9 \mathrm{deg}$ of rotation between each image that was acquired. Pixelwise fiberalignment maps were then created using Matlab 7 (The Mathworks, Inc., Natick, Massachusetts) every 0.04 s (Fig. 1), using a corresponding set of 20 images and the harmonic analysis methods described above. The loading and image acquisition rates were both chosen such that the local tissue movement during joint displacement would correspond to $<0.25$ pixels per alignment map. With tissue movement between alignment maps minimized, the fiber alignment of a single pixel could be compared between consecutive maps.

\subsection{Identifying Anomalous Fiber Realignment via Vector Correlation}

A novel analysis technique to detect microstructural damage via inferred fiber realignment was created using the correlation of consecutive alignment maps based on the premise that, when a collagen fiber breaks during loading, the local fiber network realigns as forces are redistributed to the remaining intact fibers. Vector correlation was utilized ${ }^{25,28}$ to create a robust measure of anomalous fiber realignment that included both the mean fiber direction and the strength of alignment in that mean direction (i.e., retardation). For every alignment map that was generated, vector correlation measurements were made using the maps immediately preceding and following it. A distinct correlation value was computed for each pixel in the alignment maps using just the fiber-alignment data at and surrounding that pixel. Specifically, for each pixel in the image, a correlation value was determined by comparing the data between alignment maps in a $5 \times 5$ pixel window centered at that pixel. Within that window, the pixel with the greatest vector difference between maps was removed from the correlation measurement in order to minimize the spatial propagation of error produced by any single aberrant pixel. For this correlation measure, the retardation $(\delta)$ and fiber direction $(\alpha)$ at each pixel were first converted to $x$ and $y$ components of an alignment vector. To create the alignment vector of a pixel, the axial fiber direction $(\alpha)$ was converted to circular data with an orientation of $2 \alpha$ and the magnitude of the vector was defined as $\sin ^{2}(\delta)$ to provide a linear measure of alignment strength. To compute the correlation coefficient at a pixel, two groups $(z$ and $w$ ) of alignment vectors were created from the $5 \times 5$ pixel window of the preceding and following alignment maps. The variances $\left(\sigma_{z}^{2}, \sigma_{w}^{2}\right)$ and covariance $\left(\boldsymbol{\sigma}_{z w}\right)$ of these alignment vector groups $(z$ and $w)$ were computed as follows:

$$
\sigma_{z}^{2}=\frac{1}{24} \sum_{j=1}^{24}\left(\mathbf{z}_{j}-\overline{\mathbf{z}}\right) *\left(\mathbf{z}_{j}-\overline{\mathbf{z}}\right),
$$

$$
\sigma_{w}^{2}=\frac{1}{24} \sum_{j=1}^{24}\left(\mathbf{w}_{j}-\overline{\mathbf{w}}\right) *\left(\mathbf{w}_{j}-\overline{\mathbf{w}}\right),
$$




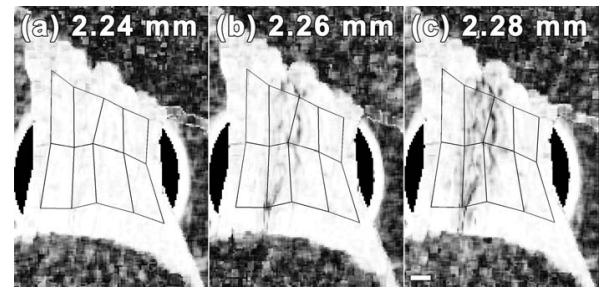

Fig. 2 Sequence of three vector correlation maps of specimen 12 during loading. The correlation coefficient at each pixel ranged from a value of 0 (black) to 1 (white) and was calculated from the surrounding fiber alignment in a $5 \times 5$ pixel window. (a) At $2.24 \mathrm{~mm}$, the vector correlation map shows almost no fiber realignment as shown by the white regions in the center of the image. (b) At $2.26 \mathrm{~mm}$, the correlation decreases in the center of the ligament midsubstance, as evident by the darker shading. (c) At $2.28 \mathrm{~mm}$, the correlation in the center of the ligament decreases even more (darker shading). Between (b) and (c), a decrease in ligament stiffness was also detected using the force-displacement data, suggesting that this decrease in vector correlation may be the result of fiber realignment associated with microstructural damage. The scale bar in (c) represents $1 \mathrm{~mm}$ and applies to all images.

$$
\boldsymbol{\sigma}_{z w}=\frac{1}{24} \sum_{j=1}^{24}\left(\mathbf{z}_{j}-\overline{\mathbf{z}}\right) *\left(\mathbf{w}_{j}-\overline{\mathbf{w}}\right),
$$

where the $x$ and $y$ coordinates of the vectors in windows $z$ and $w$ are represented in complex form, ${ }^{25}$ and $\overline{\mathbf{z}}$ and $\overline{\mathbf{W}}$ represent the mean vectors of the window in each map. The vector correlation $\left(\boldsymbol{\rho}_{z w}\right)$ between alignment maps in a given pixel window was then defined as

$$
\boldsymbol{\rho}_{z w}=\frac{\boldsymbol{\sigma}_{z w}}{\sigma_{z} \sigma_{w}} .
$$

The magnitude of this complex correlation measurement was computed to produce an analog to traditional scalar correlation values. ${ }^{25}$ This vector correlation measurement was computed for each pixel of the fiber alignment maps, and ranged from 0 to 1 , with 1 representing a consistent alignment between maps.

Using the pixelwise vector correlation calculation, correlation maps were produced for each fiber-alignment map throughout the applied loading (Fig. 2). Of note, correlation was low in any region of the image where the sample alignment was not well defined due to poor light transmission through the tissue. To identify these regions with an inadequate harmonic intensity response, pixelwise maps were also generated to quantify the signal-to-noise ratio (SNR). The root-mean-square amplitude of the harmonic response of the light intensity during polarizer rotation represented the signal. The noise was associated with the residual of the harmonic fit to the intensity response of a given pixel during the rotation of the polarizer. Pixels with an SNR of $<5$ were prone to large fluctuations in their correlation values during the continuous acquisition of fiber data in static ligaments; accordingly, any pixels with an SNR of $<5$ were removed from any further analysis.

Changes in the vector correlation between maps were quantified in order detect the anomalous fiber realignment that is proposed to occur when a collagen fiber network sustains

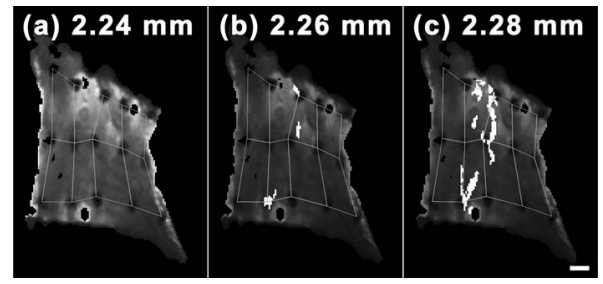

Fig. 3 Maps of anomalous fiber realignment in specimen 12 during loading. (a) From 0 to $2.24 \mathrm{~mm}$, no anomalous fiber realignment was detected for this specimen. (b) At $2.26 \mathrm{~mm}$ of displacement, fiber realignment (white pixels) was first detected in three regions in this specimen. (c) Realignment in these regions propagated into larger areas by $2.28 \mathrm{~mm}$ of displacement. Black pixels represent regions of the image where the signal-to-noise ratio was insufficient to evaluate fiber realignment. The dark gray fiduciary markers on the surface of the ligament were digitized and tracked to produce elements (outlined in gray) for strain analysis of the ligament midsubstance. The scale bar in (c) indicates $1 \mathrm{~mm}$ and applies to all images.

localized microstructural damage during loading. Anomalous fiber realignment was defined at any pixel with acceptable SNR where the vector correlation decreased by 0.2 or more relative to its correlation value in the previous correlation map (Fig. 3). A threshold of 0.2 was defined to ensure that fiber realignment would not be detected during correlation measurements made of specimens not undergoing any loading. Under the assumption that collagen fiber failure causes realignment of other fibers throughout the local fiber network, regions of tissue were defined as sustaining anomalous fiber realignment if nine or more pixels were connected to each other (based on eight-neighbor pixel connectivity). For comparison to mechanical data, the onset and location of anomalous fiber realignment were noted.

\subsection{Mechanical Data Analysis}

The average stress and strain in each specimen were calculated during loading to provide mechanical context for the onset of detected anomalous fiber realignment and to facilitate comparisons between realignment and traditional measures of tissue damage. Average ligament strain was calculated from the bright-field images in order to understand the average material response of the tissue and provide a comparison to previous mechanical studies of this ligament. Average strain was determined by digitizing and tracking the ligament insertion into the superior and inferior bone [Fig. 1(b)]. The average length between the superior and inferior insertions of the ligament was calculated from each alignment map, and the average one-dimensional (1-D) Lagrangian strain was computed throughout loading using the length measurements. The average stress, strain, force, and displacement at the first detection of anomalous fiber realignment was measured for each specimen.

To identify whether anomalous fiber realignment occurs in the same location in the tissue that undergoes the largest strains, the principal strain field on the ligament midsubstance was also calculated using the corresponding positions of the fiduciary markers. The fiduciary marker locations on the ligament midsubstance were digitized for each alignment map, and four-node elements were created from that digitized array (Figs. 2 and 3). Through isoparametric mapping and plane 
strain theory, Lagrangian strain was computed in each of these elements to provide a first principal strain field over the ligament midsubstance for every fiber-alignment map. The element with the maximum first principal strain was also noted for every alignment map during loading. Because strain could not be computed at the pixel level, the number of pixels with anomalous fiber realignment in each strain element on the ligament midsubstance was computed throughout loading to identify whether detected realignment corresponded to regions of higher tissue strains.

Gross failure, partial failure, and yield of the ligament tissue were assessed from the mechanical data collected by the Instron during loading to quantify structural damage and provide context for the occurrence of anomalous collagen fiber realignment. Gross failure was defined by the maximum force value recorded during loading. Partial failure was defined by any decrease in force with increasing displacement prior to gross failure, and yield was defined by any decrease of at least $10 \%$ in the measured tangent stiffness of the tissue. ${ }^{17,31}$ For each specimen, any occurrence of yield or partial failure that was sustained during the initial detection of fiber realignment was recorded. The onset and location of evidence of visible tissue rupture in the specimen were also defined through evaluation of the bright-field images. The criteria for the identification of visible rupture included rapid tissue movement during loading or the development of a visible hole or tear in the tissue, and the evaluator was blinded to the mechanical and polarized light data.

\subsection{Statistical Analyses}

To validate the utility of anomalous fiber realignment in identifying microstructural damage, the location of realignment was compared to visible rupture and the onset of realignment was compared to the occurrence of mechanical injury events. At the onset of visible tissue rupture, any elements were identified in the ligament's midsubstance that contained pixels that had sustained fiber realignment during loading. A two-by-two contingency table of the midsubstance elements from all specimens was created to compare those elements sustaining fiber realignment with those sustaining visible rupture, using Fischer's exact test. The displacements and average 1-D strains at each of the first detection of fiber realignment, visible rupture, and gross failure were compared using one-way ANOVAs and post-hoc Bonferroni tests. Significance for each ANOVA was defined by $p<0.05$; all tests were performed using JMP 7 (SAS Institute Inc., Cary, North Carolina).

\section{Results}

Anomalous fiber realignment was detected in 15 of the 16 specimens during tissue loading up to visible rupture. Realignment could not be detected in one specimen (No. 10) due to poor light transmission, which resulted in an insufficient SNR (Table 1). The average percentage of pixels that had sustained anomalous fiber realignment in the midsubstance elements up to the point of visible rupture was $6.2 \pm 9.0 \%$ from an average total midsubstance area of $3757 \pm 1157$ pixels. The mean thickness of these specimens was $0.451 \pm 0.092 \mathrm{~mm}$ and ranged from 0.300 to $0.632 \mathrm{~mm}$. The average percentage of pixels in the midsubstance of the ligament specimens with an acceptable SNR $(\geqslant 5)$ was
$90.7 \pm 8.4 \%$ at the beginning of loading, and the average percentage throughout the entire loading test was $84.5 \pm 12.4 \%$.

In all 15 specimens with sufficient SNR, visible rupture occurred in a region of the tissue that had sustained previous anomalous fiber realignment. Visible rupture was first detected as rapid tissue movement away from the direction of loading in 11 of the 16 specimens and as a hole in the tissue in the five other specimens. Visible rupture first occurred at $3.47 \pm 0.96 \mathrm{~mm}$ of sample displacement, which corresponded to an average 1-D strain of $1.02 \pm 0.35$ (Table 2). In six specimens, visible rupture was initially identified in the ligament midsubstance in a total of nine elements. All nine of those elements with visible rupture had sustained prior fiber realignment during loading. An additional 45 other elements (out of a 173 total elements) sustained fiber realignment in the absence of any visible rupture. The relationship between visible rupture and the localization of anomalous fiber realignment was significant $(p<0.001)$.

In every specimen, the first detection of anomalous fiber realignment coincided with the occurrence of a mechanical event that suggested some sort of tissue damage (Table 1). In 11 specimens, this realignment first occurred during the first or second occurrence of tissue failure. In three other specimens, realignment was detected during yield prior to any measurable tissue failure (Table 1). On average, fiber realignment was first detected at $2.66 \pm 0.83 \mathrm{~mm}$ of joint displacement and $15.16 \pm 9.54 \mathrm{~N}$ of load (Table 1). This corresponded to an average 1-D strain of $0.73 \pm 0.26$ and stress of 4.76 $\pm 3.11 \mathrm{MPa}$ (Table 1). The average area over which the initial fiber realignment was detected corresponded to $45.8 \pm 40.2$ pixels or $0.271 \pm 0.238 \mathrm{~mm}^{2}$ (Table 3 ). In 8 of 16 specimens, this initial realignment occurred within the elements defined on the ligament midsubstance (Table 3).

Gross mechanical failure of the specimens occurred at an average load of $21.53 \pm 7.21 \mathrm{~N}$ and displacement of $3.62 \pm 0.49 \mathrm{~mm}$. These structural data corresponded to a mean ultimate tensile stress of $6.17 \pm 2.18 \mathrm{MPa}$ and strain of $1.12 \pm 0.43$. Partial failure first occurred earlier at $15.78 \pm 9.17 \mathrm{~N}$ and $2.64 \pm 0.93 \mathrm{~mm}$, which corresponded to average stresses and strains of $4.91 \pm 3.04 \mathrm{MPa}$ and $0.72 \pm 0.27$, respectively. Ligament yield occurred at $9.48 \pm 6.11 \mathrm{~N}$ and $2.01 \pm 0.57 \mathrm{~mm}$, and corresponded to $2.93 \pm 2.01 \mathrm{MPa}$ of stress and $0.53 \pm 0.18$ strain. The mean displacement and strain at the detection of the first fiber realignment were significantly lower than both the occurrence of gross failure detected from the mechanical response ( $p$ $<0.004)$ and the first evidence of any tissue rupture evident by the bright field images $(p<0.004)$.

In the eight specimens in which initial realignment occurred in the elements in the midsubstance of the ligament, the average first principal strain of the element with the greatest number of pixels detected to have anomalous realignment was $0.32 \pm 0.27$ (Table 3 ). However, the maximum principal strain in those eight specimens at initial realignment $(0.67 \pm 0.33)$ was significantly greater $(p=0.006)$ than the first principal strain in the element with the most fiber realignment. In fact, the location of maximum principal strain only matched the element with the most fiber realignment in one specimen (No. 1; Table 3). A total of 19 elements from the eight specimens initially sustained fiber realignment; how- 
Quinn and Winkelstein: Vector correlation technique for pixel-wise detection of collagen fiber realignment...

Table 1 Summary of mechanical data at first detection of anomalous fiber realignment.

\begin{tabular}{|c|c|c|c|c|c|}
\hline Specimen & $\begin{array}{l}\text { Force } \\
(\mathrm{N})\end{array}$ & $\begin{array}{l}\text { Displacement } \\
(\mathrm{mm})\end{array}$ & $\begin{array}{l}\text { Stress } \\
(\mathrm{MPa})\end{array}$ & Strain & $\begin{array}{l}\text { Mechanical event } \\
\text { associated with } \\
\text { realignment }\end{array}$ \\
\hline 1 & 24.19 & 3.79 & 7.77 & 1.22 & 1 st failure (5th yield) \\
\hline 2 & 17.54 & 1.97 & 4.78 & 0.91 & 1 st failure (3rd yield) \\
\hline 3 & 25.94 & 3.00 & 8.42 & 0.59 & 4 th yield \\
\hline 4 & 3.74 & 1.49 & 1.90 & 0.43 & 1 st failure (1 st yield) \\
\hline 5 & 2.94 & 1.62 & 1.44 & 0.49 & 2nd failure (2nd yield) \\
\hline 6 & 13.45 & 3.43 & 2.61 & 0.65 & 7th yield \\
\hline 7 & 0.51 & 1.16 & 0.15 & 0.20 & 1 st failure (1 st yield) \\
\hline 8 & 28.39 & 2.56 & 8.08 & 0.83 & 2nd failure (3rd yield) \\
\hline 9 & 8.80 & 3.20 & 3.07 & 0.99 & 2nd failure (8th yield) \\
\hline $10^{a}$ & N/A & & & & \\
\hline 11 & 19.10 & 3.29 & 5.13 & 0.91 & 3rd failure (7th yield) \\
\hline 12 & 3.82 & 2.26 & 1.41 & 0.54 & 1 st yield \\
\hline 13 & 15.82 & 3.29 & 4.68 & 0.68 & 1 st failure (8th yield) \\
\hline 14 & 23.48 & 2.99 & 6.36 & 0.93 & 2nd failure (9th yield) \\
\hline 15 & 26.90 & 3.65 & 11.06 & 0.82 & 1 st failure (4th yield) \\
\hline 16 & 12.79 & 2.24 & 4.55 & 0.70 & 1 st failure (2nd yield) \\
\hline mean & 15.16 & 2.66 & 4.76 & 0.73 & \\
\hline S.D. & 9.54 & 0.83 & 3.11 & 0.26 & \\
\hline
\end{tabular}

ever, only three of those elements corresponded to the location of maximum principal strain for that specimen. At the onset of visible rupture, the location of a specimen's maximum principal strain matched the location of visible rupture in just three of the specimens.

\section{Discussion}

This study describes a new application of a vector correlation technique to identify changes in collagen fiber realignment in a planar ligament under tensile loading. In particular, using this method it is possible to incorporate both fiber-alignment strength and direction into an assessment of fiber realignment at each pixel during continuous loading. Specifically, using these approaches, this study found that anomalous fiber realignment in facet capsular ligament tissue is associated with an apparent loss of tissue integrity, as defined by the traditional metrics of mechanical failure, and visible rupture (Tables 1 and 2). In addition, the onset of an initial change in fiber realignment was detected well before any visible rupture of the tissue during loading (Tables 1 and 2), coinciding with either ligament yield or partial failure in every specimen
(Table 1). Additional analysis of the potential relationship between realignment and damage revealed that the spatial location where anomalous realignment was produced during loading was significantly associated with the regions in the tissue where visible rupture ultimately developed $(p<0.001)$. These findings demonstrate a method capable not only of measuring fiber realignment sensitive to mechanical trauma, but of also localizing the development of those changes with pixel resolution.

Because collagen fiber-alignment data were collected with a temporal resolution sufficient to facilitate continuous ligament loading, the onset of anomalous realignment can be directly compared to previous traditional biomechanical studies. Although the stresses and strains measured at both partial failure and gross failure in the current study are consistent with other reports for this ligament, ${ }^{8,31-33}$ these findings are the first to identify that there may be lower material limits for the capsular ligament tissue during loading (Table 1); indeed, this lower limit is based on the change in the collagen fiber realignment, which is taken as the onset of microstructural damage, ${ }^{1,2,17}$ and suggests a potential threshold for mild 
Table 2 Summary of the first detection of visible rupture.

\begin{tabular}{|c|c|c|c|c|}
\hline Specimen & $\begin{array}{l}\text { Displacement } \\
(\mathrm{mm})\end{array}$ & $\begin{array}{l}\text { Average } \\
\text { 1-D strain }\end{array}$ & $\begin{array}{l}\text { Associated } \\
\text { mechanical } \\
\text { event }\end{array}$ & Visualization criteria \\
\hline 1 & 4.53 & 1.54 & gross failure & visible hole $^{a}$ \\
\hline 2 & 3.27 & 1.76 & 5th failure & rapid tissue movement ${ }^{a}$ \\
\hline 3 & 3.64 & 0.74 & gross failure & visible hole $^{a}$ \\
\hline 4 & 1.69 & 0.50 & 2nd failure & rapid tissue movement \\
\hline 5 & 3.64 & 1.33 & gross failure & rapid tissue movement \\
\hline 6 & 3.95 & 0.78 & gross failure & rapid tissue movement ${ }^{a}$ \\
\hline 7 & 4.56 & 1.00 & gross failure & visible hole \\
\hline 8 & 2.58 & 0.84 & 2nd failure & rapid tissue movement \\
\hline 9 & 3.22 & 1.00 & 2nd failure & rapid tissue movement \\
\hline 10 & 2.85 & 0.96 & gross failure & rapid tissue movement \\
\hline 11 & 4.08 & 1.20 & gross failure & visible hole \\
\hline 12 & 2.28 & 0.55 & 1st yield & rapid tissue movement \\
\hline 13 & 5.31 & 1.24 & gross failure & rapid tissue movement \\
\hline 14 & 3.81 & 1.27 & gross failure & visible hole $^{a}$ \\
\hline 15 & 3.85 & 0.88 & gross failure & rapid tissue movement ${ }^{a}$ \\
\hline 16 & 2.26 & 0.71 & 1st failure & rapid tissue movement \\
\hline Mean & 3.47 & 1.02 & & \\
\hline S.D. & 0.96 & 0.35 & & \\
\hline
\end{tabular}

(grade I) ligament sprains ${ }^{34}$ or the development of increased joint laxity. ${ }^{3,35}$ By utilizing a detection technique with pixellevel resolution, this approach identified initial anomalous fiber realignment to occur in a region that is much smaller $\left(0.271 \pm 0.238 \mathrm{~mm}^{2}\right)$ than the size of the elements used to measure macroscale strain in this study (Fig. 3). As such, tissue strain fields may lack the sensitivity needed to detect subfailure tissue damage, which may explain the spatial disconnect between the location of maximum principal strain and the location where realignment that was first detected (Table 3 ). The implementation of the current vector correlation technique into digital image correlation techniques to estimate tissue strains may ultimately improve the accuracy of macroscale strain measurements and provide further insight into the relationship between strain and fiber realignment. Nonetheless, the findings in this study suggest the pixelwise localization of fiber realignment using vector correlation provides a more precise and accurate measure of the spatial variability in tissue injury tolerances compared to macroscale strain-field measurements from traditional light-intensity-based fiduciary marks.
The use of a pixelwise vector correlation measurement offers several advantages over other approaches to identify the fiber kinematics associated with tissue damage. Previous analysis techniques have summarized fiber direction or retardation over predefined regions of tissue or entire specimens to interpret mechanical failure. ${ }^{10,17}$ However, by combining mean fiber direction and the relative strength of fiber alignment (measured by retardation) at any given pixel into the vector correlation calculation ${ }^{25}$ [Eqs. (5)-(8)], the present analysis approach provides not only a more precise localization of fiber realignment, but also an improved sensitivity to detect changes in fiber alignment. Specifically, by assessing vector differences in this correlation technique rather than angular differences, random noise in a pixel with weak alignment is less likely than previous approaches to generate a false-positive detection of fiber realignment. ${ }^{17}$ Furthermore, unlike methods that required the complete time history of a tissue's fiber response to detect microstructural damage, ${ }^{17}$ the present technique requires only the time history of a few sequential fiber-alignment maps (in this case, acquired every $0.04 \mathrm{~s}$ ) to assess potential microstructural damage. With so 
Table 3 Summary of the localization of fiber realignment and associated strains.

\begin{tabular}{|c|c|c|c|c|c|}
\hline Specimen & $\begin{array}{l}\text { Number } \\
\text { of pixels }\end{array}$ & $\begin{array}{c}\text { Area } \\
\text { realigned } \\
\left(\mathrm{mm}^{2}\right)\end{array}$ & $\begin{array}{l}\text { Number of } \\
\text { elements with } \\
\text { realignment }\end{array}$ & $\begin{array}{l}\text { lst principal } \\
\text { strain in } \\
\text { element with } \\
\text { most } \\
\text { realignment }\end{array}$ & $\begin{array}{l}\text { Maximum } \\
\text { 1st principal } \\
\text { strain in the } \\
\text { entire } \\
\text { specimen }\end{array}$ \\
\hline 1 & 47 & 0.278 & 3 & 0.82 & 0.82 \\
\hline 2 & 16 & 0.095 & below elements & & \\
\hline 3 & 48 & 0.284 & 2 & 0.10 & 0.76 \\
\hline 4 & 17 & 0.101 & 1 & 0.07 & 0.63 \\
\hline 5 & 93 & 0.550 & right of elements & & \\
\hline 6 & 13 & 0.077 & above elements & & \\
\hline 7 & 54 & 0.320 & above elements & & \\
\hline 8 & 105 & 0.621 & 2 & 0.18 & 0.24 \\
\hline 9 & 137 & 0.811 & 5 & 0.59 & 1.32 \\
\hline $10^{a}$ & N/A & & & & \\
\hline 11 & 12 & 0.071 & above elements & & \\
\hline 12 & 74 & 0.438 & 3 & 0.36 & 0.68 \\
\hline 13 & 11 & 0.065 & below elements & & \\
\hline 14 & 9 & 0.053 & 1 & 0.35 & 0.43 \\
\hline 15 & 12 & 0.071 & above elements & & \\
\hline 16 & 39 & 0.231 & 2 & 0.13 & 0.46 \\
\hline Mean & 45.80 & 0.271 & 19 of 173 & 0.32 & 0.67 \\
\hline S.D. & 40.19 & 0.238 & & 0.27 & 0.33 \\
\hline
\end{tabular}

few fiber maps required to quantify potential microstructural damage, tissue regions that may lack sufficient light transmission during the initial portion of loading, may still be assessed for realignment if light transmission improves for a period of loading. Also, using this approach, a vector correlation map can be created between any two alignment maps in which the tissue is in the same position, regardless of the time history or map sequence. Although a QPLI system provides the microstructural data in this study, this vector correlation technique could be adapted to other imaging modalities, such as secondharmonic-generation microscopy or optical coherence tomography ${ }^{12,14,36}$ capable of measuring fiber alignment in an in vivo setting. Ultimately, an assessment of anomalous fiber realignment during tissue loading in vivo could provide unique insight into the relationship between microstructural injury and potential physiologic consequences of such injuries. ${ }^{4,22,34}$

Using quantitative polarized light imaging, fiber alignment can be measured continuously anywhere in a planar, collagenous soft tissue. Accordingly, changes to the collagen fiber network, and possibly damage, can be evaluated by transmit- ting light through the total area of any given tissue sample. However, accurate fiber measurements may be limited to ligament samples with thicknesses of $<0.6 \mathrm{~mm}$, given the occasional poor light transmission in the thickest samples measured in this study. Although application of this technique may be limited by sample thickness, the surface area of entire specimens could be accommodated in this study by adjusting the camera field of view. As a result, the magnification was insufficient to actually visualize the putative collagen fiber failures during loading that may occur when fiber realignment is detected. As such, analysis of tissue integrity via electron microscopy ${ }^{37,38}$ would be needed in the ligament regions where anomalous realignment was detected in order to provide ultrastructural validation of the central premise that collagen fiber failure mediates an atypical pattern of fiber realignment. Likewise, because anomalous fiber realignment is presumed to occur in the local fiber network surrounding fiber failure, the specific location(s) of collagen fiber failure within the detected anomalous realignment is unknown. Therefore, the true extent and severity of damage cannot be fully distin- 
guished through this imaging technique. Furthermore, it remains unclear whether the fiber realignment detected in this study is permanent or unrecoverable. Additional investigations are needed to assess if there are any sustained structural and mechanical effects of loading up to the onset of anomalous fiber realignment. Although mechanical detection of tissue yield or failure coincided with the initial detection of fiber realignment in every test, realignment was not detected during or before the first occurrence of partial failure in 5 of the 16 specimens (Table 1). To conclusively determine the accuracy of the vector correlation technique for detecting the onset of microstructural damage, additional work is needed to determine if damage is actually produced in the soft tissue at every incidence of a failure or yield.

Nonetheless, with pixelwise damage detection, the vector correlation-based analysis technique provides a substantial advancement in the ability to identify and localize microstructural kinematics associated with potential tissue damage during loading. Because this polarized light analysis technique detects anomalous fiber realignment during injurious loading, it cannot serve as a clinical diagnostic tool. Rather, it provides an experimental framework to understand how the microstructural composition of biological tissue can give rise to region-specific mechanical thresholds of tissue injury. Ultimately, by detecting the true onset of mechanically induced injury, we can begin to understand how tissue loading can produce mechanical changes and physiologic dysfunction in the absence of visible rupture.

\section{Acknowledgments}

This work was funded by grants from the Association for the Advancement of Automotive Medicine and the Catharine D. Sharpe Foundation. This material is based on work supported by the National Science Foundation under Grant No. 0547451.

\section{References}

1. J. A. Gimbel, J. P. Van Kleunen, S. Mehta, S. M. Perry, G. R. Williams, and L. J. Soslowsky, "Supraspinatus tendon organizational and mechanical properties in a chronic rotator cuff tear animal model," $J$. Biomech. 37(5), 739-749 (2004).

2. K. P. Quinn, K. E. Lee, C. C. Ahaghotu, and B. A. Winkelstein, "Structural changes in the cervical facet capsular ligament: potential contributions to pain following subfailure loading," Proc. Stapp Car Crash Conf. 51, 169-187 (2007).

3. P. P. Provenzano, D. Heisey, K. Hayashi, R. Lakes, and R. Vanderby, Jr., "Subfailure damage in ligament: a structural and cellular evaluation," J. Appl. Physiol. 92(1), 362-371 (2002).

4. Y. Lu, C. Chen, S. Kallakuri, A. Patwardhan, and J. M. Cavanaugh, "Neurophysiological and biomechanical characterization of goat cervical facet joint capsules," J. Orthop. Res. 23(4), 779-787 (2005).

5. N. Yoganandan, J. F. Cusick, F. A. Pintar, and R. D. Rao, "Whiplash injury determination with conventional spine imaging and cryomicrotomy," Spine 26(22), 2443-2448 (2001).

6. P. J. Macmahon, S. Dheer, S. M. Raikin, I. Elias, W. B. Morrison, E. C. Kavanagh, and A. Zoga, "MRI of injuries to the first interosseous cuneometatarsal (Lisfranc) ligament," Skeletal Radiol. 38(3), 255260 (2009).

7. M. A. Kliewer, L. Gray, J. Paver, W. D. Richardson, J. B. Vogler, J. H. McElhaney, and B. S. Myers, "Acute spinal ligament disruption: MR imaging with anatomic correlation," J. Magn. Reson Imaging 3(6), 855-861 (1993).

8. G. P. Siegmund, B. S. Myers, M. B. Davis, H. F. Bohnet, and B. A. Winkelstein, "Mechanical evidence of cervical facet capsule injury during whiplash: a cadaveric study using combined shear, compression, and extension loading," Spine 26(19), 2095-2101 (2001).
9. F. R. Noyes and E. S. Grood, "The strength of the anterior cruciate ligament in humans and Rhesus monkeys," J. Bone Jt. Surg., Am. Vol. 58(8), 1074-1082 (1976).

10. T. T. Tower, M. R. Neidert, and R. T. Tranquillo, "Fiber alignment imaging during mechanical testing of soft tissues," Ann. Biomed. Eng. 30(10), 1221-1233 (2002).

11. P. S. Robinson and R. T. Tranquillo, "Planar biaxial behavior of fibrin-based tissue-engineered heart valve leaflets," Tissue Eng. Part $A$ (in press).

12. K. A. Hansen, J. A. Weiss, and J. K. Barton, "Recruitment of tendon crimp with applied tensile strain," J. Biomech. Eng. 124(1), 72-77 (2002).

13. J. G. Snedeker, G. Pelled, Y. Zilberman, A. Ben Arav, E. Huber, R. Muller, and D. Gazit, "An analytical model for elucidating tendon tissue structure and biomechanical function from in vivo cellular confocal microscopy images," Cells Tissues Organs 190(2), 111119(2009).

14. J. G. Snedeker, G. Pelled, Y. Zilberman, F. Gerhard, R. Muller, and D. Gazit, "Endoscopic cellular microscopy for in vivo biomechanical assessment of tendon function," J. Biomed. Opt. 11(6), 064010 (2006).

15. K. L. Billiar and M. S. Sacks, "A method to quantify the fiber kinematics of planar tissues under biaxial stretch," J. Biomech. 30(7), 753-756 (1997).

16. M. A. Geday, W. Kaminsky, J. G. Lewis, and A. M. Glazer, "Images of absolute retardance L. Deltan, using the rotating polariser method," J. Microsc. 198(Pt 1), 1-9 (2000).

17. K. P. Quinn and B. A. Winkelstein, "Altered collagen fiber kinematics define the onset of localized ligament damage during loading," $J$. Appl. Physiol. 105(6), 1881-1888 (2008).

18. A. Gefen, B. van Nierop, D. L. Bader, and C. W. Oomens, "Straintime cell-death threshold for skeletal muscle in a tissue-engineered model system for deep tissue injury," J. Biomech. 41(9), 2003-2012 (2008).

19. B. Deng, P. C. Begeman, K. H. Yang, S. Tashman, and A. I. King, "Kinematics of human cadaver cervical spine during low speed rearend impacts," Proc. Stapp Car Crash Conf. 44, 171-188 (2000).

20. H. L. Cater, L. E. Sundstrom, and B. Morrison, "Temporal development of hippocampal cell death is dependent on tissue strain but not strain rate," J. Biomech. 39(15), 2810-2818 (2006).

21. A. C. Bain and D. F. Meaney, "Tissue-level thresholds for axonal damage in an experimental model of central nervous system white matter injury," J. Biomech. Eng. 122(6), 615-622 (2000).

22. K. E. Lee, J. H. Thinnes, D. S. Gokhin, and B. A. Winkelstein, "A novel rodent neck pain model of facet-mediated behavioral hypersensitivity: implications for persistent pain and whiplash injury," J. Neurosci. Methods 137(2), 151-159 (2004).

23. T. T. Tower and R. T. Tranquillo, "Alignment maps of tissues: II. fast harmonic analysis for imaging," Biophys. J. 81(5), 2964-2971 (2001).

24. T. T. Tower and R. T. Tranquillo, "Alignment maps of tissues: I. microscopic elliptical polarimetry,” Biophys. J. 81(5), 2954-2963 (2001).

25. B. Hanson, K. Klink, K. Matsuura, S. M. Robeson, and C. J. Willmott, "Vector correlation: review, exposition, and geographic application," Ann. Assoc. Am. Geogr. 82(1), 103-116 (1992).

26. A. P. Sviridov, Z. Ulissi, V. Chernomordik, M. Hassan, and A. H. Gandjbakhche, "Visualization of biological texture using correlation coefficient images," J. Biomed. Opt. 11(6), 060504 (2006).

27. D. Zhang and D. D. Arola, "Applications of digital image correlation to biological tissues," J. Biomed. Opt. 9(4), 691-699 (2004).

28. P. Kaufmann and R. O. Weber, "Directional correlation coefficient for channeled flow and application to wind data over complex terrain," $J$. Atmos. Ocean. Technol. 15(1), 89-97 (1998).

29. P. P. Provenzano, A. L. Alejandro-Osorio, W. B. Valhmu, K. T. Jensen, and R. Vanderby, Jr., "Intrinsic fibroblast-mediated remodeling of damaged collagenous matrices in vivo," Matrix Biol. 23(8), 543-555 (2005).

30. A. M. Glazer, J. G. Lewis, and W. Kaminsky, “An automatic optical imaging system for birefringent media," Proc. R. Soc. London, Ser. A 452(1955), 2751-2765 (1996).

31. K. P. Quinn and B. A. Winkelstein, "Cervical facet capsular ligament yield defines the threshold for injury and persistent joint-mediated neck pain," J. Biomech. 40(10), 2299-2306 (2007).

32. N. Yoganandan, S. Kumaresan, and F. A. Pintar, "Geometric and 
mechanical properties of human cervical spine ligaments," J. Biomech. Eng. 122(6), 623-629 (2000).

33. B. A. Winkelstein, R. W. Nightingale, W. J. Richardson, and B. S. Myers, "The cervical facet capsule and its role in whiplash injury: a biomechanical investigation," Spine 25(10), 1238-1246 (2000).

34. L. Jones, Q. Bismil, F. Alyas, D. Connell, and J. Bell, "Persistent symptoms following non operative management in low grade MCL injury of the knee-the role of the deep MCL," The Knee 16(1), 64-68 (2009).

35. M. M. Panjabi, E. Yoldas, T. R. Oxland, and J. J. Crisco, 3rd, "Subfailure injury of the rabbit anterior cruciate ligament," J. Orthop. Res. 14(2), 216-222 (1996).
36. S. Psilodimitrakopoulos, S. I. Santos, I. Amat-Roldan, A. K. Thayil, D. Artigas, and P. Loza-Alvarez, "In vivo, pixel-resolution mapping of thick filaments' orientation in nonfibrilar muscle using polarization-sensitive second harmonic generation microscopy," $J$. Biomed. Opt. 14(1), 014001 (2009).

37. E. A. Sander and V. H. Barocas, "Comparison of 2D fiber network orientation measurement methods," J. Biomed. Mater. Res. Part A 88(2), 322-331 (2009).

38. P. P. Provenzano and R. Vanderby, Jr., "Collagen fibril morphology and organization: implications for force transmission in ligament and tendon," Matrix Biol. 25(2), 71-84 (2006). 